\title{
As mães sabem avaliar adequadamente o peso das crianças?
}

\section{Do mothers know how to evaluate infant weight?}

Maria Antonieta de Barros Leite CARVALHAES'

Ilda de GODOY ${ }^{1}$

\section{RE S U M O}

Investigou-se a adequação da opinião materna sobre o peso de crianças menores de dois anos, submetidas à monitorização do crescimento em unidades básicas de saúde. Foram entrevistadas 180 mães e, com base nas respostas, anotadas na íntegra e codificadas a posteriori, as crianças foram classificadas em desnutridas ou em risco, eutróficas ou com sobrepeso. Esta classificação foi comparada com a condição nutricional dos infantes, definida de acordo com os critérios antropométricos adotados pelos serviços de saúde. Segundo os resultados, como instrumento para o diagnóstico precoce da desnutrição e do sobrepeso, a opinião materna apresentou baixa sensibilidade e alta especificidade. A freqüência de adequação do parecer das mães não se relacionou com a idade, escolaridade e posição da mãe em relação ao trabalho. Conclui-se que a maior parte das mães de crianças com problemas relacionados à alimentação não apresentava condições para participar ativamente das ações de prevenção/recuperação por desconhecerem o estado nutricional de seus filhos.

Termos de indexação: monitorização do crescimento, educação em saúde, assistência primária à saúde, avaliação nutricional, criança.

\section{A B S T R A C T}

This paper investigated how adequate maternal opinion was about the nutritional status of children under two years of age, who had their growth monitored in health care units. One hundred and eighty mothers were interviewed and, based on their responses, written down in full and codified a posteriori, the children were classified as underweight, normal weight and overweight. This classification was compared with the nutritional status of the infants, determined in accordance with anthropometric criteria used by the health services. According to the results, maternal opinion, as a tool for the early diagnosis of malnutrition and overweight, showed low sensitivity and high specificity. Socioeconomic factors - mother's educational status, age and

\footnotetext{
1 Departamento de Enfermagem, Faculdade de Medicina de Botucatu, Universidade Estadual Paulista. Distrito de Rubião Jr, s/n, 18618-970, Botucatu, SP, Brasil. Correspondência para/Correspondence to: M.A.B.L. CARVALHAES. E-mail: carvalha@fmb.unesp.br
} 
156 | M.A.B.L. CARVALHAES \& I. GODOY

working position - were not associated with the adequacy of the opinions. It was concluded that the majority of the mothers of children with food problems were not able to actively participate in actions of recovery/ prevention because they did not know the infants nutritional status.

Index terms: growth monitoring, health education, primary health care, nutrition assessment, child.

\section{NTRODUÇÃ O}

A monitorização do crescimento foi proposta no início da década de setenta como atividade central da assistência primária à saúde infantil (World Health..., 1978). Desde então, detectar déficits de crescimento tem sido considerada a maneira mais eficaz para o diagnóstico precoce de problemas de saúde e nutrição nos primeiros anos de vida (Batista Filho \& Rissin, 1993). Para o Fundo das Nações Unidas para a Infância (UNICEF), um dos incentivadores de projetos visando esta prática em todos os países em desenvolvimento, quando realizada com o envolvimento materno, esta atividade permite à mãe adotar medidas adequadas de cuidado, influindo favoravelmente sobre o estado de saúde e nutrição da criança (United Nations..., 1989).

Avaliações de diversos programas de intervenção nutricional os quais implementaram a monitorização do crescimento a partir da década de oitenta mostraram que seus objetivos não estavam sendo facilmente alcançados.

Gopalan \& Chatterjee (1985) analisaram as experiências da Índia, Indonésia e Filipinas em projetos para efetuar este tipo de acompanhamento. Seus resultados evidenciaram falhas na operacionalização, tais como erros dos trabalhadores de saúde ao medir e anotar o peso nos gráficos, balanças descalibradas e problemas ainda mais graves, como a equivocada interpretação da curva de crescimento e o desconhecimento das medidas a serem adotadas diante dos "déficits" constatados. A participação das mães também foi considerada bastante insatisfatória.

Gerein \& Ross (1991) acompanharam três programas de saúde desenvolvidos no Zaire, nos quais a monitorização do crescimento foi utilizada para selecionar crianças em risco. O estudo identificou que a monitorização do crescimento era insuficientemente utilizada para aconselhamento das mães e na adoção de medidas dirigidas à recuperação ou prevenção da desnutrição.

De acordo com Nabarro \& Chinnock (1988), para ser uma ação de saúde eficaz, é preciso implementar adequadamente esta monitorização, o que inclui a correta tomada e anotação das medidas nos gráficos de crescimento. Além disso, com maior importância, há a necessidade de os trabalhadores de saúde e as mães compreenderem claramente seu significado e saberem quais atitudes adotar diante do crescimento insuficiente.

De fato, após realizar revisão da literatura disponível na década de oitenta, Lotfi (1988) encontrou indícios de efeitos positivos desta prática, como redução da mortalidade e da severidade dos episódios de diarréia, queda nas taxas de desnutrição e aumento da capacidade materna de avaliar a saúde infantil. Entretanto, os estudos apresentavam dificuldade de isolar o componente monitorização do crescimento das demais ações ligadas à saúde e à sobrevivência infantil.

O debate sobre a eficácia da monitorização do crescimento permaneceu durante a década de noventa. Recentemente, Garner et al. (2000) desenvolveram uma acurada revisão dos estudos com delineamento experimental ou quasi-experimental (ensaio clínico) os quais avaliaram os efeitos desta atividade em termos de medidas antropométricas, conhecimentos e satisfação materna, mortalidade e morbidade na infância. Encontraram apenas dois trabalhos que 
preencheram os critérios estabelecidos para inclusão na pesquisa, embora nenhum tenha medido seu alcance relativamente à morbi-mortalidade. No primeiro, realizado em áreas rurais da Índia, foram comparados dois grupos participantes de um projeto de assistência primária à saúde, dos quais apenas um foi submetido à monitorização do crescimento. Não foram detectadas diferenças entre os dois grupos em relação ao ganho de peso durante 30 meses. No entanto, os autores não descartaram a possibilidade de este resultado ser devido ao pequeno número de indivíduos pesquisados, aspecto que reduziu o poder estatístico do estudo. O segundo ensaio analisado pelos pesquisadores acima referidos foi desenvolvido em Lesotho. A intervenção incluiu a pesagem rotineira das crianças e um trabalho educativo sobre a interpretação de dois modelos de curva de crescimento. Foram avaliados os conhecimentos maternos antes e após quatro meses da intervenção. Os resultados indicaram melhora significativa nos escores de conhecimento, sendo as diferenças maiores no grupo onde empregou-se um determinado tipo de curva, denominado "caminho da saúde". Segundo os autores da revisão, não há evidências conclusivas quanto ao efeito da monitorização do crescimento sobre o ganho de peso. Quanto ao componente educativo, há demonstrações claras de melhora na capacidade materna de avaliar a saúde infantil após sua participação em projetos incentivadores desta prática.

Senanayake et al. (1997); Meegan \& Morley (1999) detectaram progresso significativo na capacidade materna de avaliar o estado nutricional da criança quando a monitorização do crescimento foi implementada com forte caráter educativo, incluindo o emprego de curvas adaptadas para facilitar a compreensão das mães sobre seu significado, sendo uma delas acoplada à própria balança.

No Brasil, de acordo com pesquisa desenvolvida em Santa Catarina sobre o desempenho da Pastoral da Criança, organismo de ação social ligado à igreja católica que inclui entre suas diversas atividades relacionadas à saúde a monitorização do crescimento com participação materna, revelou que as mães assistidas apresentavam escores mais altos em teste realizado para avaliar sua capacidade de interpretar curvas de crescimento indicativas de desnutrição crônica e aguda (Neumann et al., 1999).

Não foram encontrados registros sobre operacionalização, impacto ou conhecimentos maternos relativos ao acompanhamento do crescimento desenvolvido nas unidades básicas de saúde do Estado de São Paulo desde o início da década de oitenta (São Paulo..., 1985).

Neste artigo, são apresentados os resultados de um levantamento sobre a concordância entre a opinião materna e a classificação nutricional de crianças submetidas rotineiramente à monitorização do crescimento em unidades básicas de saúde da rede pública, localizadas na área urbana de município da Região Centro-Sul do Estado de São Paulo. Este estudo integra um projeto mais amplo direcionado para a investigação da participação materna nas atividades básicas da assistência primária à saúde infantil. Considera-se o envolvimento da mãe uma característica necessária para que a monitorização do crescimento possa produzir impacto positivo sobre a saúde da criança (Organización Panamericana..., 1981).

\section{CASUISTICAE METODOS}

Trata-se de um estudo transversal, no qual foi avaliada uma amostra das 860 crianças menores de dois anos matriculadas em seis das oito unidades básicas de saúde (UBS) existentes na área urbana do município em 1995. Nas UBS inseridas na pesquisa, a monitorização do crescimento estava sendo desenvolvida há mais de dois anos, sem interrupção. Em consultas periódicas agendadas, o peso era medido e anotado no prontuário e no gráfico de crescimento 
nele incluído por auxiliares de enfermagem, cabendo ao médico (ou ao enfermeiro) sua interpretação e a tomada das medidas adequadas à condição nutricional da criança.

Foram estudadas 180 mães, e o número de entrevistadas em cada UBS foi proporcional ao total de crianças matriculadas em cada uma destas unidades, variando de 10 a 70. As mães foram selecionadas mediante sorteio, dentre aquelas que aguardavam consulta agendada. A fase de seleção e a coleta de dados durou até completar-se o número de entrevistas programado em cada unidade de saúde.

As entrevistas foram efetuadas por alunos do $4^{\circ}$ ano da graduação em enfermagem, previamente treinados. As mães foram abordadas após a realização do atendimento (consulta médica ou de enfermagem) que motivou sua ida ao serviço de saúde e convidadas a participar do estudo. Obteve-se autorização da Secretaria Municipal de Saúde do município e do Comitê de Ética em Pesquisa da Faculdade de Medicina de Botucatu para a realização deste trabalho.

A opinião das mães foi obtida com uma questão aberta: "a sra. poderia me contar sua opinião sobre o peso de seu filho?" As respostas, anotadas na íntegra, foram codificadas a posteriori pelo pesquisador principal. Considerou-se a criança com desnutrição ou risco de desnutrição, conforme julgamento materno, quando as declarações incluíram conceitos como "magrinho", "fraquinho", "miúdo demais", "não engorda/ cresce de jeito nenhum", "pequeno para a idade", "menor/mais leve" que crianças da mesma idade e outros semelhantes. Quando a mãe referiu termos como "peso bom", "peso normal", "nem gordo nem magro", "está bom para a idade" e outros similares, seu parecer foi classificado como criança eutrófica. Avaliações nas quais observaram-se expressões como "pesa demais", "está acima do normal", "é muito gordo", foram classificadas como criança com sobrepeso/ obesidade. Além da opinião das mães, foram obtidos dados socioeconômicos, relativos à salubridade do ambiente, e demográficos, com vistas à caracterização da população estudada.

A classificação nutricional da criança segundo a opinião materna foi comparada ao seu estado nutricional, definido com base no peso medido no dia da entrevista e classificado de acordo com os critérios adotados pelas UBS estudadas: peso menor que o percentil 10, criança desnutrida ou em risco; peso igual ou superior ao percentil 10 e inferior ao percentil 90, criança eutrófica; e peso igual ou acima do percentil 90, criança com sobrepeso/obesidade. Estes critérios foram preconizados por Monteiro (1984) e adotados pela Secretaria de Estado da Saúde de São Paulo em 1985, vigorando nas unidades de saúde pesquisadas (São Paulo..., 1985).

Avaliou-se a freqüência de concordância entre as duas classificações e calculou-se a sensibilidade e a especificidade da "opinião materna" para o diagnóstico precoce de desvios do estado nutricional, de acordo com Almeida Filho \& Rouquayrol (1992). Como critério "verdade" ou "ouro" considerou-se o vigente nas unidades de saúde, acima referido.

A seguir, investigou-se a relação entre a adequação da opinião da mãe e as variáveis idade, escolaridade e exercício de trabalho fora do domicílio. As associações pesquisadas foram submetidas a teste de significância estatística $\left(\chi^{2}\right)$, utilizando-se $p<0,05$ como nível crítico. Os dados foram armazenados e analisados com o software Epi Info 5.0.

\section{RESULTADOS}

As mães entrevistadas tinham baixa escolaridade - $61 \%$ tinham até a $4^{\text {a }}$ série concluída, viviam em domicílios com boas condições de saneamento, $25 \%$ delas eram adolescentes, apenas um quinto delas trabalhava fora de casa (Tabela 1).

Verificou-se os seguintes resultados: 15,0\% das crianças foram consideradas desnutridas por suas mães; $81,6 \%$, eutróficas; 3,4\%, com 
sobrepeso/obesidade (Tabela 2). Na Tabela 3, encontra-se a distribuição das crianças segundo a classificação nutricional baseada na opinião materna e aquela de acordo com os critérios já referidos. Pode-se notar que as mães identificaram corretamente $36,7 \%$ das crianças desnutridas, $87,4 \%$ das eutróficas e apenas $13,6 \%$ daquelas com sobrepeso/obesidade.

Tabela 1. Características socioeconômicas e demográficas das mães. Unidades Básicas de Saúde, Botucatu, 1995.

\begin{tabular}{|c|c|c|}
\hline Variáveis & $n$ & $\%$ \\
\hline \multicolumn{3}{|l|}{ Escolaridade } \\
\hline Até $4^{\mathrm{a}}$ série do $1^{\circ} \mathrm{grau}$ & 109 & 60,9 \\
\hline De $5^{\mathrm{a}}$ a $8^{\mathrm{a}}$ série do $1^{\circ} \mathrm{grau}$ & 44 & 24,6 \\
\hline A partir da $1^{\mathrm{a}}$ série do $2^{\circ}$ grau & 26 & 14,5 \\
\hline \multicolumn{3}{|l|}{ Abastecimento de água } \\
\hline Adequado (água tratada, ponto dentro de casa) & 163 & 91,1 \\
\hline Inadequado & 16 & 8,9 \\
\hline \multicolumn{3}{|l|}{ Banheiro } \\
\hline Adequado (com descarga, ligado à rede ou fossa) & 156 & 87,2 \\
\hline Inadequado & 23 & 12,8 \\
\hline \multicolumn{3}{|l|}{ Pessoas no dormitório com a criança (inclusive) } \\
\hline Menor ou igual a 3 & 137 & 77,0 \\
\hline Maior que 3 & 42 & 23,0 \\
\hline \multicolumn{3}{|l|}{ Idade materna (anos) } \\
\hline Menor que 20 & 44 & 24,6 \\
\hline Maior ou igual a 20 & 135 & 75,4 \\
\hline \multicolumn{3}{|l|}{ Trabalho da mãe (fora de casa) } \\
\hline Sim & 39 & 21,8 \\
\hline Não & 140 & 78,2 \\
\hline
\end{tabular}

Tabela 2. Opinião das mães sobre o peso de seus filhos. Unidades Básicas de Saúde, Botucatu, 1995.

\begin{tabular}{lcc}
\hline Opinião materna & $\mathrm{n}$ & $\%$ \\
\hline Desnutrição & 27 & 15,28 \\
Eutrofia & 146 & 81,56 \\
Sobrepeso/obesidade & 6 & 3,16 \\
\hline Total & 179 & 100,00 \\
\hline
\end{tabular}

Comparando-se os critérios "opinião materna" e "antropometria adotada nos serviços de saúde estudados" o primeiro apresentou baixa sensibilidade (36,7\%), alta especificidade $(89,3 \%)$ e baixo valor de predição positivo $(40,7 \%)$ no diagnóstico precoce da desnutrição. Para o diagnóstico do sobrepeso, o desempenho foi ainda pior: sensibilidade de $13,6 \%$, especificidade de $96,8 \%$ e valor de predição positivo de 50,0\%.

Os resultados não mostraram diferenças significativas, isto é, as freqüências de mães que acertaram ou erraram a condição nutricional da criança não variaram em relação à idade e escolaridade materna. Também não foi encontrada associação entre adequação da opinião materna e trabalho fora de casa (Tabela 4).

\section{DISCUSSÃO}

De acordo com este estudo, 63,3\% das mães das crianças com déficit de peso (peso abaixo do percentil 10) não reconheceram esta condição. Da mesma forma, $86,4 \%$ das mães das crianças com sobrepeso não identificaram o excesso de peso de seus filhos. Estes resultados revelam baixo envolvimento materno na monitorização do crescimento de seus filhos. Como o conhecimento adequado da situação nutricional da criança é considerado a primeira condição para a ativa participação das mães, mediante ações de cuidado, na prevenção de problemas ou na recuperação nutricional, pode-se afirmar que nas unidades de saúde avaliadas um dos principais objetivos da monitorização do crescimento não estava sendo alcançado (Organización Panamericana..., 1981).

Tabela 3. Distribuição das crianças segundo opinião da mãe sobre seu peso e classificação nutricional de acordo com critério das UBS(s), Botucatu, 1995.

\begin{tabular}{|c|c|c|c|c|c|c|c|c|}
\hline \multirow{3}{*}{ Classificação nutricional } & \multicolumn{8}{|c|}{ Opinião das mães } \\
\hline & \multicolumn{2}{|c|}{ Desnutrição } & \multicolumn{2}{|c|}{ Eutrofia } & \multicolumn{2}{|c|}{ Sobrepeso } & \multicolumn{2}{|c|}{ Total } \\
\hline & $n$ & $\%$ & $\mathrm{n}$ & $\%$ & $\mathrm{n}$ & $\%$ & $\mathrm{n}$ & $\%$ \\
\hline Desnutrição & 11 & 36,7 & 19 & 63,3 & - & - & 30 & 100,0 \\
\hline Eutrofia & 13 & 10,2 & 111 & 87,4 & 3 & 2,4 & 127 & 100,0 \\
\hline Sobrepeso & 3 & 13,6 & 16 & 72,7 & 3 & 13,6 & 22 & 100,0 \\
\hline
\end{tabular}


Tabela 4. Freqüência de mães que acertaram e erraram a classificação nutricional da criança segundo idade materna, escolaridade e trabalho fora do domicílio. Unidades Básicas de Saúde, Botucatu, 1995.

\begin{tabular}{|c|c|c|c|c|c|c|}
\hline \multirow{2}{*}{ Variável } & \multicolumn{2}{|c|}{ Acertaram } & \multicolumn{2}{|c|}{ Erraram } & \multicolumn{2}{|c|}{ Total } \\
\hline & $\mathrm{n}$ & $\%$ & $n$ & $\%$ & $n$ & $\%$ \\
\hline \multicolumn{7}{|l|}{ Idade materna* } \\
\hline $19 \leq$ & 32 & 72,7 & 12 & 27,3 & 44 & 100,0 \\
\hline $20-30$ & 64 & 67,3 & 31 & 32,7 & 95 & 100,0 \\
\hline $31 \geq$ & 24 & 60,0 & 16 & 40,0 & 40 & 100,0 \\
\hline \multicolumn{7}{|l|}{ Escolaridade $^{* *}$} \\
\hline até 4 série do $1^{\circ} \mathrm{grau}$ & 78 & 71,6 & 31 & 28,4 & 109 & 100,0 \\
\hline $5^{\mathrm{a}}$ a $8^{\mathrm{a}}$ série do $1^{\circ} \mathrm{grau}$ & 28 & 63,6 & 16 & 36,4 & 44 & 100,0 \\
\hline$\geq 1^{\text {a }}$ série do $2^{\circ}$ grau & 19 & 73,0 & 7 & 24,0 & 26 & 100,0 \\
\hline \multicolumn{7}{|l|}{ Trabalho Materno*** } \\
\hline Sim & 25 & 64,1 & 14 & 35,9 & 39 & 100,0 \\
\hline Não & 100 & 71,2 & 10 & 28,6 & 140 & 100,0 \\
\hline
\end{tabular}

${ }^{*} \chi^{2}=1,55 ; p>0,05$ (N.S.); ${ }^{* *} \chi^{2}=1,085 ; p>0,05$ (N.S.); ${ }^{* *} \chi^{2}=0,778 ; p>0,05$ (N.S.).

Quais seriam os fatores responsáveis pela situação observada? As mães incluídas nesta pesquisa eram predominantemente de baixa escolaridade e esta característica pode dificultar a compreensão do significado do peso como indicador nutricional (Cleland \& Van Ginneken, 1988). Poder-se-ia, também, supor que as adolescentes, menos experientes e preparadas para o cuidado infantil, apresentassem maior freqüência de opiniões inadequadas. Porém, não houve diferenças significativas de concordância em relação às variáveis idade e grau de escolaridade das mães.

Outro fator com possível influência negativa sobre a capacidade materna de avaliar o peso da criança seria o trabalho fora do domicílio, o qual, por exemplo, poderia reduzir o número de comparecimentos no serviços de saúde (Bennet,1988). Também não houve, neste estudo, associação entre este fator e a adequação das avaliações maternas.

Existe ainda a possibilidade de algumas mães terem recebido informações divergentes da avaliação efetuada segundo os critérios em vigor nas UBS. Crianças com déficits leves de peso podem ter sido consideradas eutróficas pelos profissionais que as assistiam, levando-se em consideração sua altura, tamanho dos pais, história clínica, peso ao nascimento, entre outros fatores. De fato, adotando-se o percentil 10 de peso para a idade como ponto de corte para diagnóstico precoce da desnutrição, é possível um certo percentual de falsos positivos, tanto maior quanto menor o número de crianças expostas à desnutrição (Monteiro, 1984).

Para verificar esta possibilidade, testou-se o critério materno apenas para as crianças com peso para a idade abaixo do percentil 3, ponto de corte capaz de detectar formas moderadas e severas de desnutrição. A sensibilidade do critério "opinião materna" aumentou, mas ainda foi insatisfatória: $50 \%$.

A monitorização do crescimento não foi concebida para o diagnóstico precoce do sobrepeso/obesidade nos primeiros anos de vida. Entretanto, com a transição nutricional em curso em nosso meio e o aumento do risco de ocorrência destes problemas, inclusive na infância, maior atenção deverá ser dada a esta finalidade. Conforme indicam os dados obtidos nesta pesquisa, as mães tendem a não reconhecer o sobrepeso nas crianças.

Em síntese, os resultados do presente estudo podem ser explicados pela ausência ou inadequação de atividades educativas visando a participação das mães na monitorização do 
crescimento. De fato, constatou-se que o principal instrumento educativo desta prática - a cópia do gráfico de controle do crescimento impressa no verso da carteira de vacinação da criança, isto é, o chamado cartão da criança, não era utilizado nas unidades de saúde estudadas. As cópias permaneciam em branco. Apenas as curvas de crescimento dos prontuários estavam preenchidas. Já foi apontado, em diversas partes do mundo, que a monitorização do crescimento, com ênfase na educação, tem sido melhor implementada em projetos comunitários do que em unidades de assistência à saúde (Morley, 1994; Meegan \& Morley, 1999).

Conclui-se que as mães, sem ações educativas especialmente dirigidas para o seu envolvimento no acompanhamento nutricional, têm capacidade limitada de utilizar o peso da criança como indicador precoce de desvios nutricionais.

\section{A GRADECIMENTOS}

As autoras agradecem aos alunos das Disciplinas "Enfermagem em Saúde Pública" e "Nutrição e Dietética aplicada à Enfermagem" pela realização das entrevistas e digitação dos dados.

\section{REFERENCIAS BIBLIOGRAFICAS}

ALMEIDA FILHO, N., ROUQUAYROL, M.Z. Introdução à epidemiologia moderna. 2.ed. Belo Horizonte : Abrasco, 1992. p.29-44.

BATISTA FILHO, M.S., RISSIN, A. Deficiências nutricionais: ações específicas do setor saúde para seu controle. Cadernos de Saúde Pública, Rio de Janeiro, v.9, n.2, p.30-35,1993.

BENNETT, L. The role of women in income prodution and intra-household allocation of resources as a determinant of child nutrition and health. Food and Nutrition Bulletin, Tokyo, v.10, n.3, p.16-26, 1988.
CLELAND, I.G., VAN GINNEKEN, J.K. Maternal education and child survival in developing countries: the search for pathways of influence. Social Science and Medicine, Oxford, v.27, n.12, p.1357-1368, 1988.

GARNER, P., PANPANICH, R., LOGAN, S. Is routine growth monitoring effective? A systematic review of trials. Archives of Disease in Childhood, London, v.82, n.3, p.197-201, 2000.

GEREIN, N., ROSS, D.A. Is growth monitoring worthwhile? An evaluation of its use in three child health programmes in Zaire. Social Science and Medicine, Oxford, v.32, n.6, p.667-675,1991.

GOPALAN, C., CHATTERJEE, M. Use of growth charts for promoting child nutrition: a review of global experience. Delhi : Nutrition Foundation of India, 1985. (Special Publications Serie, 2).

LOTFI, M. Growth monitoring: a brief literature review of current knowledge. Food and Nutrition Bulletin, Tokyo, v.10, n.4, p.3-10, 1988.

MEEGAN, M., MORLEY, D.C. Growth monitoring: family participation: effective community development. Tropical Doctor, London, v.29, p.23-27, 1999.

MONTEIRO, C.A. Critérios antropométricos no diagnóstico da desnutrição em programas de assistência à criança. Revista de Saúde Pública, São Paulo, v.18, n.3, p.209-217, 1984.

MORLEY, D. Will growth monitoring continue to be part of primary health care? South African Medical Journal, Cape Town, p.15-16, 1994. Supplement.

NABARRO, D., CHINNOCK, P. Growth monitoring: inappropriate promotion of an appropriate technology. Social Science and Medicine, Oxford, v.26, n.9, p.941-948, 1988.

NEUMANN, N.A., VICTORA, C.G., HALPERN, R., GUIMARÃES, P.R.V., CESAR, J.A. Desempenho da Pastoral da Criança na promoção de ações de sobrevivência infantil e na educação em saúde em Crisciúma, uma cidade do Sul do Brasil. Revista Panamericana de la Salud, v.5, n.6, p.400-410, 1999.

ORGANIZACIÒN PANAMERICANA DE LA SALUD. Ficha de crecimiento para uso internacional en el cuidado 
162 | M.A.B.L. CARVALHAES \& I. GODOY

de la salud materna e infantil: guia para el personal de Atención Primaria de Salud. Washington DC, 1981. 38p. (Publicación Científica, n.409).

SÃO PAULO. Secretaria de Estado da Saúde. Normas para o acompanhamento do crescimento e desenvolvimento da criança. São Paulo : Departamento Técnico Normativo, Divisão de Normas Técnicas, 1985. 12p. (Norma Técnica S.S., n.32/85). (Mimeografado).

SENANAYAKE, M.P., GUNAWARDENA, M.K.S., PEIRIS, D.S.P. Maternal comprehension of two growth monitoring charts in Sri Lanka. Archives of
Disease in Childhood, London, v.76, n.4, p.359-361, 1997.

UNITED NATIONS CHILDREN'S FUND. Estratégia para melhorar a nutrição de crianças e mulheres nos países em desenvolvimento: um exame de políticas. New York, 1989. 38p.

WORLD HEALTH ORGANIZATION. A growth chart for international and child health care: guidelines for primary health care personal. Geneva, 1978. 36p.

Recebido para publicação em 22 de setembro de 2000 e aceito em 11 de junho de 2001. 